\title{
Si deve aerare, aereare o areare un ambiente? L'importante è ventilarlo!
}

\author{
Paolo D'Achille
}

PUBBLICATO: 28 APRILE 2020

\section{Quesito:}

Sono arrivate varie domande, dislocate nel tempo, che ci chiedono spiegazioni sulle diverse varianti di uno stesso verbo: c'e chi domanda se le forme aereare e areare siano erronee, chi segnala che la forma aerare, indicata come la più corretta nei principali dizionari, sia in realtà più rara delle altre due, chi vorrebbe sapere quali sono le varianti piu antiche.

\section{Si deve aerare, aereare o areare un ambiente? Limportante è ventilarlo!}

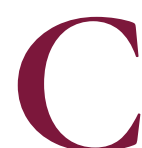

on questa triade di varianti si ripropone, in termini diversi, una questione già trattata da Luca Serianni nel primo numero della "Crusca per voi" (ottobre i99o, p. 9) e riprodotta nel nostro sito il 3I gennaio 2003, a proposito della correttezza di aeroplano e aeroporto rispetto ad areoplano, areoporto. Tutte queste voci, come varie altre, sono infatti riconducibili a una stessa "famiglia di parole", che ha come "capostipite" il latino aèr genitivo aèris "aria' (a sua volta dal greco aér, genitivo aéros): si veda al riguardo il recente volume dell'ASLI Scuola, Repertorio Italiano di Famiglie di parole. Dagli etimi ai significati per arricchire il lessico, a cura di Michele Colombo e Paolo D’Achille, Bologna, Zanichelli, 2019, p. I5.

Cominciamo col confermare che la forma oggi considerata più corretta è aerare, che sincronicamente si può considerare deverbale da aere (dal latino aěre $(m)$, accusativo di $a \bar{e} r$ ), a cui si aggiungono direttamente le desinenze dei verbi della prima classe verbale (che ha l'infinito in-are). Più probabilmente, in prospettiva diacronica, il verbo deriva dal corrispondente francese aérer, che risulta attestato già nel Trecento. Ma la sequenza vocalica ae costituisce uno iato non ammesso nel tradizionale sistema tosco-fiorentino, tanto che, accanto ad aere, parola dotta, esiste l'allotropo popolare aria, tratto dal latino aěra, un'altra forma, modellata sul greco, dell'accusativo di aér, con successiva metatesi (cioè scambio di posizione di due foni). Così, nel parlato, si è diffusa la pronuncia popolare areare, anch'essa con metatesi (e forse favorita dall'esistenza della parola area 'misura della superficie'), che si trova non di rado anche nello scritto (ma che vari dizionari, come lo Zingarelli 2020, registrano come forma da evitare). La terza variante, aereare, registrata nei dizionari senza censure ma considerata rara, si spiega con accostamento all'aggettivo aereo 'dell'aria' (dal latino aĕrĭu $(m)$, derivato anch'esso da aēr), da cui deriva pure, per conversione, il nome dell'aereo, usato come sinonimo di aeroplano (e che è alla base di forme come aereoplano, aereoporto).

Come nel caso di aerare, anche i sostantivi aerazione e aeratore (da considerare, in sincronia, deverbali del verbo, ma anch'essi, in diacronia, forse modellati sui precedenti francesi aération e aérateur) hanno come varianti, rispettivamente, aerazione, aereazione, e aereatore, areatore, che si spiegano nello stesso modo.

I1 DELI data il verbo aerare al I9Io (e non offre indicazioni cronologiche sulle varianti), mentre fa risalire le prime attestazioni delle forme aereazione e aereatore rispettivamente al I867 e al I942, anteriormente alle varianti aerazione e aeratore, datate rispettivamente i919 e 1965; anche l'aggettivo aereato $\left(\mathrm{I}_{783}\right)$ precede aerato ( 1788 ); entrambi, in ogni caso, sarebbero più antichi del verbo. Il 
GRADIT data invece aerare al I875, aerazione al I884 e aeratore al I942, e cosi, negli ultimi due casi, lo Zingarelli, che però per aerare risale al 1784 (prima, dunque di aerato e aereato, il che autorizza a considerare l'aggettivo come conversione dal corrispondente participio).

Una ricerca su Google libri consente, per il verbo, di documentare entro il sec. XVIII anche la forma aereare, che è attestata in una Memoria del medico Gioseffantonio Dardana (Vercelli, Panialis, I79o, p. 27), in cui, in una nota a commento di un aereato del testo, si legge: "(b) Sotto il verbo aereare e i suoi derivati io non intendo in questa memoria, se non se dar aria, renderne il suo corso libero, ventilarla, rinnovarla ec.; non altro senso, spezialmente chimico". Quanto ad areare, è documentato nella Breve istruzione per evitare il danno che reca il calcino o mal del segno ai filugelli e per governare nel miglior modo questi utili animaletti (Milano, Molina, I839, p. 29), in un passo in cui si danno consigli "per liberare i bachi dal calcino, di porli in locali meno caldi e più areati... Si procuri di areare la stanza più che si può"; il passo è citato nel "Giornale agrario lombardo-veneto", XV, I84I, p. 337, che aggiunge, a riprova di una censura incipiente della forma, "(Volevasi dire aereati e aererare)".

Lo stesso motore di ricerca permette di risalire ai primi dell'Ottocento per aereazione, che è documentato nei Fondamenti della scienza fisico-chimica applicati alla formazione dei corpi ed ai fenomeni della natura di Vincenzo Dandolo ( $6^{\mathrm{a}}$ ed., vol. I, Venezia, Pasquali, I802, p. 231), e precede di pochi anni aerazione, che risulta attestato per la prima volta nella traduzione (di Antonio Targioni Tozzetti) degli Elementi di chimica agraria in un corso di lezioni per il pensionato di agricoltura di Humphry Davy (Firenze, Piatti, vol. II, I8I5, p. I2). Anche la forma areazione, oggi censurata, è ben documentata nel corso dell'Ottocento: il primo esempio che ho reperito è in un articolo nei "Nuovi annali delle scienze naturali”, serie II, tomo I, I844, p. 4I8.

Quanto ad aeratore, è attestato in Beniamino Caso, Studi su le attuali pratiche agrarie nella provincia di Capitanata e sul modo di migliorarle (Torino, Unione Tipografica-editrice, i869, p. 50; ancora precedente è la segnalazione del "Ventilatore-aeratore dei signori fratelli Mazzellini" nel "Giornale dell'ingegnere-architetto ed agronomo", V, I857, pp. 222 e 346). Allo stesso anno risale aereatore, usato come aggettivo (granaio aereatore), in un articolo edito su "L'Italia agricola", I, I869, p. 36, mentre la variante areatore, seguita nella prima occorrenza dalla glossa tra parentesi (assorbitore d'aria), si trova in L'uso ed il maneggio delle caldaie e macchine navali di Viktor H. Sirk, edizione italiana a cura di Federico Rosenberg (Torino, Loescher, I877, p. 229).

In definitiva, la documentazione raccolta consente di verificare come le diverse varianti siano pressappoco coeve e tutte abbiano un retroterra storico considerevole, in testi non di matrice popolare. Oggi, come si è detto all'inizio, le forme considerate standard sono aerare, aeratore, aerazione, che hanno dalla loro la vicinanza col confisso aero-, ma la difficoltà fonetica da un lato e la prossimità anche semantica con l'aggettivo aereo dall'altro fanno si che tanto areare, areatore, areazione (che non costituiscono, comunque, delle banalizzazioni recenti e che nel parlato non accurato sono forse quelle più usate), quanto soprattutto aereare, aereatore, aereazione, non siano affatto uscite dall'uso. Nello scritto, in ogni caso, consigliamo di attenersi alle scelte e alle indicazioni, sostanzialmente concordi, dei vari dizionari.

\section{Cita come:}

Paolo D'Achille, Si deve aerare, aereare o areare un ambiente? L'importante è ventilarlo! , "Italiano digitale", 2019, XIII, 2020/2 (aprile-giugno)

DOI: $10.35948 / 2532-9006 / 2020.3323$

Copyright 2019 Accademia della Crusca

Pubblicato con licenza creative commons CC BY-NC-ND 